\title{
Vertical structuring of gigantic jets
}

\author{
Caitano L. da Silva ${ }^{1}$ and Victor P. Pasko ${ }^{1}$ \\ Received 6 May 2013; revised 28 May 2013; accepted 29 May 2013; published 20 June 2013.
}

[1] Gigantic Jets (GJs) are initiated deep inside the thundercloud as intracloud discharges whose upward-directed leaders manage to escape through the thundercloud top and propagate up to the ionosphere. The speed at which leaders propagate is limited by the air heating of every newly formed leader section, rate of which is slower at upper altitudes in the Earth's atmosphere. Despite the expected deceleration of an upward-directed leader, GJs are observed to accelerate as they approach the ionosphere. In this letter, we discuss the dependence of the leader speed on current density in the leader stem, and we propose a simple timedynamic model for GJ propagation that includes the effects of the expansion of the streamer zone adjacent to the leader head. We propose that the GJ acceleration is a consequence of its vertical structuring and, therefore, can be used to trace the transition altitude between the leader and streamer zone sections of GJs. Citation: da Silva, C. L., and V. P. Pasko (2013), Vertical structuring of gigantic jets, Geophys. Res. Lett., 40, 3315-3319, doi:10.1002/grl.50596.

\section{Introduction}

[2] Gigantic jets (GJs) are upward-directed large-scale electrical discharges that are observed to leave thundercloud tops and propagate up to $\sim 90 \mathrm{~km}$ altitude, connecting to the ionosphere [Pasko et al., 2002; Su et al., 2003]. GJs are the most recently discovered member of the Transient Luminous Events (TLEs) family. The first scientific recording of a GJ was obtained in 2001 [Pasko et al., 2002]. The observations were made from the Arecibo Observatory in Puerto Rico, and the GJ occurred over an oceanic thunderstorm cell $\sim 200 \mathrm{~km}$ away from the observation site. Pasko et al. [2002] have estimated the bright portions of the GJ to reach altitudes as high as $\sim 70 \mathrm{~km}$; however, further analysis demonstrated that the terminal altitude of the observed GJ was $\sim 84 \mathrm{~km}$ [da Silva and Pasko, 2012, Figure 1a]. Pasko et al. [2002] have observed the GJ to emerge from the thundercloud top at a speed $\sim 5 \times 10^{4} \mathrm{~m} / \mathrm{s}$ and to accelerate, reaching a speed $\gtrsim 10^{6} \mathrm{~m} / \mathrm{s}$ [see also da Silva and Pasko, 2012, Figure 3a]. The term "gigantic jet" was introduced by Su et al. [2003]. These authors have observed five GJs emerging from an oceanic thunderstorm near the Philippines and reaching altitudes $\sim 86-91 \mathrm{~km}$.

${ }^{1}$ CSSL, Department of Electrical Engineering, Pennsylvania State University, University Park, Pennsylvania, USA.

Corresponding author: C. L. da Silva, Communications and Space Sciences Laboratory, Department of Electrical Engineering, Pennsylvania State University, 227 EE East, University Park, PA 16802-2706, USA. (caitano.dasilva@psu.edu)

(C)2013. American Geophysical Union. All Rights Reserved. 0094-8276/13/10.1002/grl.50596
Su et al. [2003] pointed out the existence of three phenomenologically distinct stages in the observed GJs: the leading jet stage corresponding to the upward propagation, the fully developed jet stage with persistent luminosity after the connection with the ionosphere, and the trailing jet corresponding to the lower part of the GJ that decays slower than other portions. Su et al. [2003] also reported that GJs persisted $\sim 417-650 \mathrm{~ms}$ and that average velocities were $\sim 1-1.2 \times 10^{6} \mathrm{~m} / \mathrm{s}$.

[3] In recent years, the number of ground-based [Cummer et al., 2009; van der Velde et al., 2010; Soula et al., 2011; Lu et al., 2011] and satellite-based [Kuo et al., 2009, and references therein] observations of GJs has increased considerably. Remote-sensing of VLF emissions have revealed that most GJs are of negative polarity and transport hundreds of coulombs of negative charge to the ionosphere [e.g., Cummer et al., 2009]. The current theoretical understanding of the GJ process describes it as an upward-directed discharge, analogous to cloud-to-ground lightning [Krehbiel et al., 2008]. In a normal-polarity thunderstorm (i.e., containing a midlevel negative and an upper positive charge centers), GJs are initiated between adjacent charge regions (similarly to intracloud lightning discharges), where the electric field is the highest [Krehbiel et al., 2008]. Lightning is initiated by a bidirectional discharge that propagates in the form of positive leaders in the negative charge region and in the form of negative leaders in the positive charge region [e.g., Riousset et al., 2007]. Krehbiel et al. [2008] demonstrated that when the two charges were not balanced (meaning the upper positive charge center contains less net charge than the midlevel negative charge center), the leader potential could be significantly shifted in the direction defined by the charge with dominant magnitude. In this situation the propagation of the leader becomes essentially independent from the weaker charge center, allowing it to penetrate through the weaker upper charge center and to escape from the thundercloud upward and serve as the initiation of a GJ [Krehbiel et al., 2008].

[4] Complementarily, Raizer et al. [2006] point out that as the leader propagates upward, the streamer zone ahead of it becomes longer, because of the dynamics of streamer growth in a medium with exponentially decreasing air density. Therefore, there is an altitude where the streamer corona in the leader head can "escape" to the ionosphere. In the present work, we present results of a streamer-to-leader transition model capable of describing the leader formation and propagation in a broad range of ambient air density encompassing the altitude range of GJs [da Silva and Pasko, 2012]. We present a simple time dynamic model for the description of GJ propagation, and finally, we explain the vertical structuring of GJs by combining results of our time-dynamic model with the ideas introduced by Raizer et al. [2006] and Krehbiel et al. [2008]. 


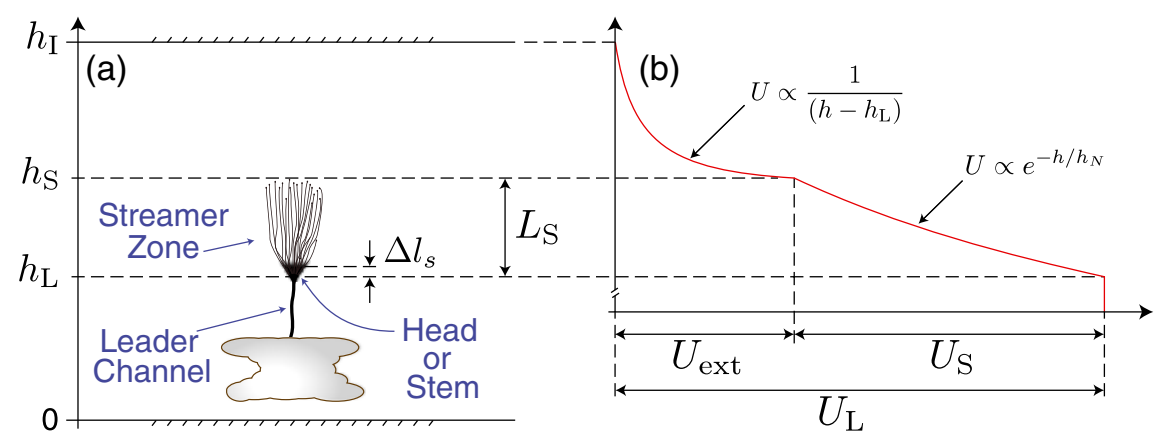

Figure 1. (a) Sketch of GJ upward propagation defining length scales discussed in text. (b) Sketch of electric potential drop from the leader head to the ionosphere.

\section{Modeling of Leader Speeds}

[5] It is well accepted that the leader speed is dictated by the air heating in every newly formed portion of the elongating leader [e.g., Bazelyan and Raizer, 2000, pp. 66-67]. For this reason, both experimental [e.g., Andreev et al., 2008] and theoretical [e.g., Popov, 2009] studies attempt to provide leader speed as a function of electrical current flowing through the leader head into the channel, i.e., $v_{\mathrm{L}}=v_{\mathrm{L}}(I)$, instead of any other parameter, such as electric potential, for example. The theoretical approach for estimation of leader speed is to assume that a constant current $I$ is flowing through the leader stem and to calculate the time $\tau_{\mathrm{h}}$ to heat the stem up to $\sim 2000 \mathrm{~K}$. When temperature reaches this threshold the formation of a highly conducting new section of the leader is unavoidable [Popov, 2009]. The streamer-to-leader transition takes place on a time scale $\tau_{\mathrm{h}}$ at which the leader extends a distance $\Delta l_{s}$ in space. Therefore, leader speed can be estimated as $v_{\mathrm{L}}=\Delta l_{s} / \tau_{\mathrm{h}}$ [e.g., da Silva and Pasko, 2012, equation (1)]. The leader streamer zone is a conically shaped fan of thousands of streamers [e.g., Bazelyan and Raizer, 2000, Figure 2.11]. Figure 1a illustrates this structuring in the context of GJs. The length of the conducting section behind the tips of individual streamers is $\Delta l_{\mathrm{s}}=v_{\mathrm{s}} \tau_{\mathrm{a} 3}$, where $v_{\mathrm{s}}$ is the streamer velocity and $\tau_{\mathrm{a} 3}$ is the three-body electron attachment time scale. For a streamer velocity $v_{\mathrm{s}} \simeq 10^{5} \mathrm{~m} / \mathrm{s}$ (typical of young weak streamers) and for $\tau_{\mathrm{a} 3} \simeq 10^{-7} N_{0}^{2} / N^{2} \mathrm{~s}$, it gives $\Delta l_{\mathrm{s}} \simeq 1 N_{0}^{2} / N^{2} \mathrm{~cm}$ [da Silva and Pasko, 2012, and references therein], where $N_{0}$ and $N$ are air densities of ground level and altitude of interest, respectively. This size of $\Delta l_{s}$ is comparable with the measured radius of the leader head in laboratory discharges at ground pressure [Bazelyan et al., 2007]. Therefore, one can suppose that the leader head, which is clearly visible on laboratory photographs (and streak images), is a collection of initial, still conducting, closely located streamer segments [Bazelyan et al., 2007]. In the present work, streamer properties at a given altitude $h$ in the Earth's atmosphere are obtained by scaling the respective value at ground-level air density $N_{0}$ to the corresponding value at reduced air density $N(h)$, following similarity laws for streamer physics [e.g., Pasko, 2006, pp. 265-267], where $N(h)=N_{0} e^{-h / h_{N}}$, with $h_{N}=7.2 \mathrm{~km}$ and $N_{0}=2.5 \times 10^{19} \mathrm{~cm}^{-3}$. We note that threebody attachment is a very inefficient plasma decay process at mesospheric altitudes $\left(N \ll N_{0}\right)$. Hence, the assumption that the streamer channel lifetime is dictated by $\tau_{\mathrm{a} 3}$ is not correct at sprite altitudes. However, the concept of $\Delta l_{\mathrm{s}} \propto \tau_{\mathrm{a} 3}$ is only used here to estimate leader speeds below $\sim 30 \mathrm{~km}$ altitude, as shown in Figure 2.

[6] In order to calculate the streamer-to-leader transition timescale, we have developed a model that simulates the air heating process in the leader stem. The model is built on previous work by Riousset et al. [2010] and accounts for the Joule heating of air through the so-called fast heating mechanism, as well as vibrational excitation of nitrogen molecules and its delayed relaxation into translational energy. The initial results were presented in a previous publication [da Silva and Pasko, 2012], where the initial radial distribution of the electron density $n_{e}=n_{e, a} e^{-r^{2} / r_{c}^{2}}$ in the leader stem was assumed to resemble a single streamer, with $n_{e, a}=$ $2 \times 10^{14} N^{2} / N_{0}^{2} \mathrm{~cm}^{-3}$ and $r_{c}=0.3 N_{0} / N \mathrm{~mm}$. Using the above mentioned value for $r_{c}$ we obtained a dependence $v_{\mathrm{L}}(I)$ [da Silva and Pasko, 2012, Figure 2b]. The parameterization of leader speed with respect to the electrical current $I$ flowing throug the channel is a common approach used in literature [e.g., Popov, 2009], because channel base current is a parameter easily obtainable from experiments [e.g., Andreev et al., 2008]. However, from a physical standpoint, the leader speed should be more generally defined as a function of the current density $J$ rather than the total current $I$. Figures $2 \mathrm{a}$ and $2 \mathrm{~b}$ present simulated leader speed as a function of the initial current density in the leader stem, at ground $\left(\Delta l_{s}=1 \mathrm{~cm}\right)$ and $20 \mathrm{~km}$ altitude $\left(\Delta l_{s}=2.1 \mathrm{~m}\right)$, respectively. We note that current density scales with air density as $\propto N^{2}$ and the range of current values shown in Figures $2 \mathrm{a}$ and $2 \mathrm{~b}$ are different by a factor of 200 , approximately reflecting this scaling. We can see a similar dependence on $J=I / \pi r_{c}^{2}$ for both altitudes and for a one order of magnitude range of change in $r_{c}$. We can also see that the same leader speed can be obtained with two orders of magnitude difference in I. The value $r_{c}=0.3 \mathrm{~mm}$ has been proven to accurately reproduce the characteristics of laboratory leaders, which are generated in meter-long gaps, under potential differences of hundreds of kilovolts to a few megavolts [e.g., Popov, 2009]. Under these conditions, the leader has $I \sim 1 \mathrm{~A}$ and $v_{\mathrm{L}} \sim 10^{4}$ $\mathrm{m} / \mathrm{s}$ [Bazelyan and Raizer, 2000, p. 67]. However, in the formation of a leader in open air with available thundercloud potential, the initial radius for the stem might be significantly larger due to various reasons, for example, streamer expansion and overlapping.

[7] The two quantities that determine leader speed are $\tau_{\mathrm{h}}$ and $\Delta l_{s}$, and they are dictated by air heating and threebody attachment, respectively. Time scale for both processes 

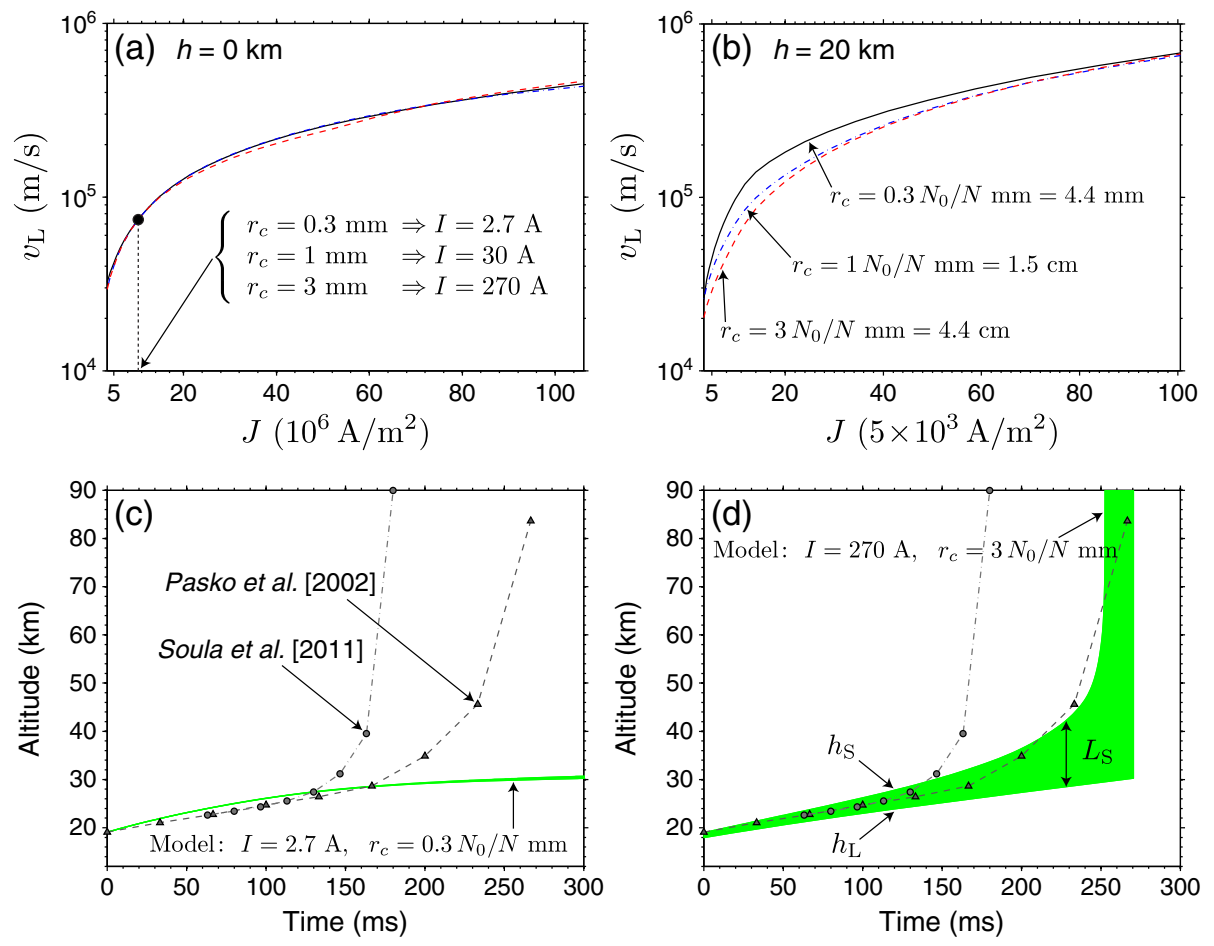

Figure 2. Simulated leader speed as a function of initial current density in the leader stem at (a) ground and (b) 20 $\mathrm{km}$ altitude, for different values of stem radius. Comparison of observed GJ propagation with modeled upward leader propagation for $J=9.6 \times 10^{6} N^{2} / N_{0}^{2} \mathrm{~A} / \mathrm{m}^{2}$, including expansion of streamer zone, for two different values of stem radius, (c) $0.3 \mathrm{~mm}$ and (d) $3 \mathrm{~mm}$.

increases with reducing air density as $\propto 1 / N^{2}$, therefore, leader speed presents weak dependence on ambient air density, as also shown in Figures 2a and 2b. Although we do not discuss details of the different dynamical features of positive and negative leaders, we assume that the streamer-to-leader transition is a fundamental process that defines leader propagation in both cases. In the case of a negative leader, this process occurs during the growth of a space leader ahead of the main leader channel. The growth of the space leader is the slowest process in the sequence of relatively fast events accompanying development of a stepped leader, and we assume that in time average sense, it is the main process defining speed with which the negative leader advances in space [da Silva and Pasko, 2012, and references therein].

\section{Expansion of GJ Streamer Zone}

[8] Theory of leader discharges predicts the existence of an average constant electric field in the streamer zone equal to the critical electric field value for stable streamer propagation $E_{\mathrm{cr}}$ [Bazelyan and Raizer, 2000, pp. 67-69]. For a positive leader at ambient ground pressure, for example, this value is $E_{\mathrm{cr}, 0} \simeq 5 \mathrm{kV} / \mathrm{cm}$ [Bazelyan and Raizer, 2000, p. 69]. For the sake of simplicity, most of the calculations in this paper are performed for positive leaders. We also include some representative values for negative leaders by introducing the critical electric field for negative streamer propagation, which is $12.5 \mathrm{kV} / \mathrm{cm}$, at ground pressure [e.g., Pasko, 2006, p. 261, and references therein]. The average electric field in a leader streamer zone is expected to reduce exponentially with altitude proportionally to air density, i.e., $E_{\mathrm{cr}}=E_{\mathrm{cr}, 0} N / N_{0}[$ Pasko, 2006, p. 266]. As first noticed by
Raizer et al. [2006], this fact has important consequences in an upward propagating leader, such as in the case of GJs escaping from thundercloud tops. Consider an upwardpropagating leader with its head located at an altitude $h_{\mathrm{L}}$, as sketched in Figure 1a. The leader head carries a potential $U_{\mathrm{L}}$ with respect to the ionosphere, located at an altitude $h_{\mathrm{I}}=90 \mathrm{~km}$. The potential drop between the leader head and ionosphere can be written as follows:

$$
U_{\mathrm{L}}=U_{\mathrm{S}}+U_{\mathrm{ext}},
$$

where $U_{\mathrm{S}}$ is the potential drop in the streamer zone $\left(h_{\mathrm{L}}<h<\right.$ $h_{\mathrm{S}}=h_{\mathrm{L}}+L_{\mathrm{S}}$, where $L_{\mathrm{S}}$ is the length of the streamer zone) and $U_{\text {ext }}$ is the potential drop in the remaining region between the top of the streamer zone and the ionosphere $\left(h_{\mathrm{S}}<h<h_{\mathrm{I}}\right)$, as sketched in Figure 1b. The potential drop in the streamer zone is as follows [Raizer et al., 2006, equation (3)]:

$$
U_{\mathrm{S}}=\int_{h_{\mathrm{L}}}^{h_{\mathrm{S}}} E_{\mathrm{cr}, 0} e^{-h / h_{N}} \mathrm{~d} h=h_{N}\left(E_{\mathrm{cr}, \mathrm{L}}-E_{\mathrm{cr}, \mathrm{S}}\right),
$$

where $E_{\mathrm{cr}, \mathrm{L}}=E_{\mathrm{cr}, 0} e^{-h_{\mathrm{L}} / h_{N}}$ and $E_{\mathrm{cr}, \mathrm{S}}=E_{\mathrm{cr}, 0} e^{-h_{\mathrm{S}} / h_{N}}$. The potential drop in the remaining portion can be calculated by assuming that the electric field is continuous through the boundary of the streamer zone, and that it is proportional to $1 /\left(h-h_{\mathrm{L}}\right)^{2}$ [Bazelyan and Raizer, 2000, p. 69]. Such electric field can be produced by a point charge $Q_{\mathrm{L}}=4 \pi \varepsilon_{0} L_{\mathrm{S}}^{2} E_{\mathrm{cr}, \mathrm{S}}$ placed at altitude $h_{\mathrm{L}}$. Therefore, the remaining potential drop is as follows:

$$
U_{\mathrm{ext}}=\int_{h_{\mathrm{S}}}^{h_{\mathrm{I}}} E_{\mathrm{cr}, \mathrm{S}}\left(\frac{L_{\mathrm{S}}}{h-h_{\mathrm{L}}}\right)^{2} \mathrm{~d} h=L_{\mathrm{S}} E_{\mathrm{cr}, \mathrm{S}}\left(\frac{h_{\mathrm{I}}-h_{\mathrm{S}}}{h_{\mathrm{I}}-h_{\mathrm{L}}}\right) .
$$



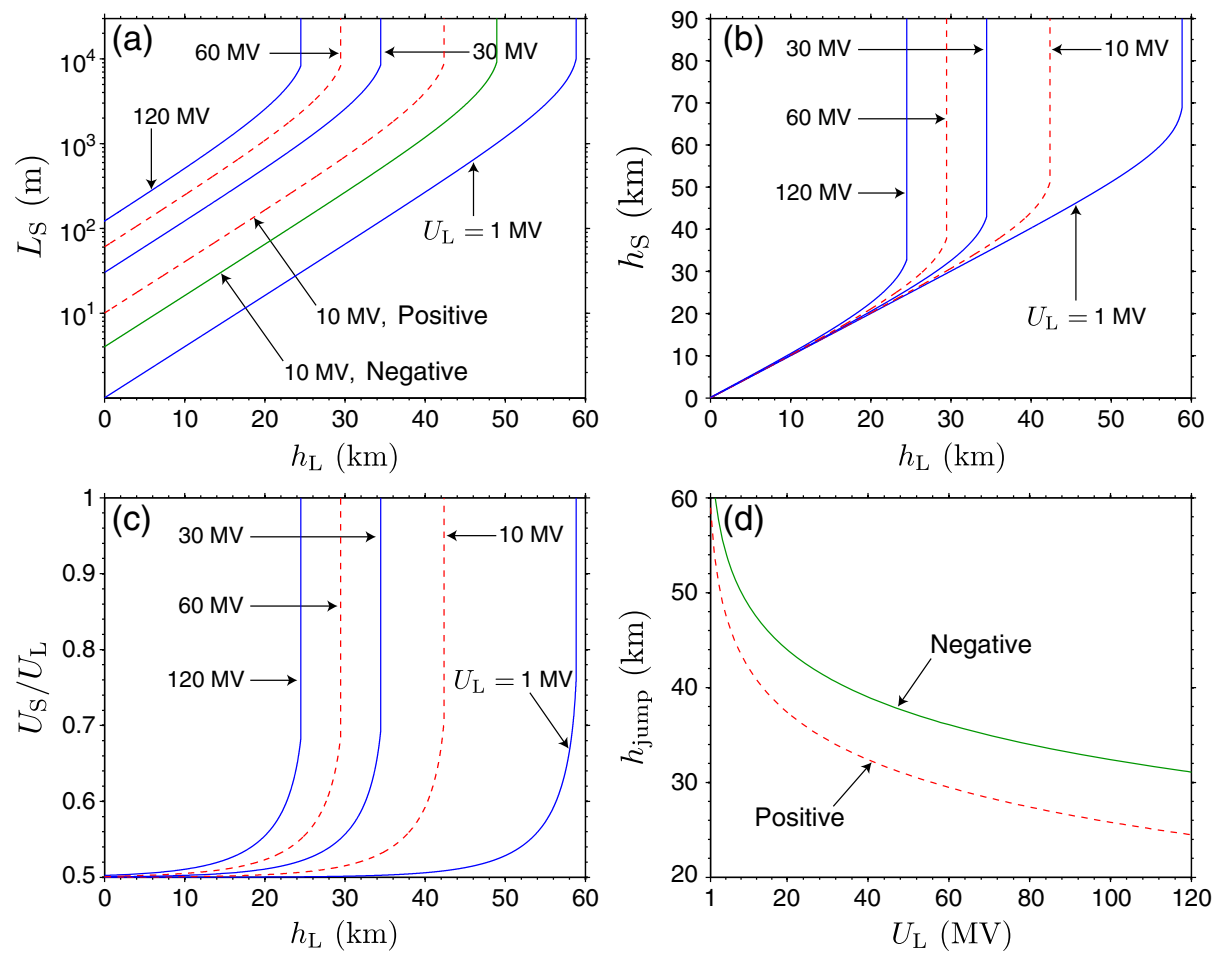

Figure 3. Calculated (a) streamer zone length $L_{\mathrm{S}}$ and (b) top altitude $h_{\mathrm{S}}=h_{\mathrm{L}}+L_{\mathrm{S}}$ as functions of leader head altitude $h_{\mathrm{L}}$. (c) Fraction of potential drop across the streamer zone $U_{\mathrm{S}} / U_{\mathrm{L}}$. (d) Altitude at which streamer zone jumps to the ionosphere $h_{\text {jump }}$ as a function of $U_{\mathrm{L}}$. Presented values correspond to positive leaders unless otherwise indicated.

For simplicity, effects of all other charges in the system are ignored.

[9] Figures $3 \mathrm{a}$ and $3 \mathrm{~b}$ present the calculated length of the streamer zone $L_{\mathrm{S}}$ and top altitude $h_{\mathrm{S}}$, respectively, by numerically inverting equation (1). It is evident from the figures that owing to the exponential decrease in air density, the size of streamer zone increases with altitude location of leader head $h_{\mathrm{L}}$. Moreover, there is an altitude $h_{\text {jump }}$ so that indefinite streamer growth is possible, and the streamer zone becomes so long that it "jumps" to the ionosphere. To further illustrate these relationships, we discuss limit solutions for equations (1)-(3). When $L_{\mathrm{S}}$ is small $\left(L_{\mathrm{S}} \ll h_{N}\right.$ and $\left.h_{\mathrm{S}} \ll h_{\mathrm{I}}\right)$ we have $U_{\mathrm{S}}=U_{\text {ext }}=L_{\mathrm{S}} E_{\mathrm{cr}, \mathrm{L}}$. This leads to the classical result that half of the leader voltage drop occurs in the streamer zone and to the well-known formula for its length $L_{\mathrm{S}}=U_{\mathrm{L}} / 2 E_{\mathrm{cr}, \mathrm{L}}$ [Bazelyan and Raizer, 2000, p. 69], which provides the initial slope in curves presented in Figure 3a. In the other extreme, when the streamer zone approaches the ionosphere $h_{\mathrm{S}} \rightarrow h_{\mathrm{I}}, U_{\mathrm{ext}} \rightarrow 0$ and, consequently, $U_{\mathrm{L}} \approx U_{\mathrm{S}}$. Figure $3 \mathrm{c}$ presents the ratio $U_{\mathrm{S}} / U_{\mathrm{L}}$ as a function of $h_{\mathrm{L}}$ to illustrate that the potential drop in the streamer zone shifts from $U_{\mathrm{L}} / 2$ to $U_{\mathrm{L}}$ as the leader propagates upward and approaches the jump altitude. Thus, we can obtain an analytical approximation for the length of the streamer zone, by inverting equation (2) [Raizer et al., 2006, eq. (4)]:

$$
L_{\mathrm{S}}=h_{N} \ln \left[\left(1-\frac{U_{\mathrm{S}}}{h_{N} E_{\mathrm{cr}, \mathrm{L}}}\right)^{-1}\right],
$$

where $U_{\mathrm{S}}=U_{\mathrm{L}} / 2$ if $h_{\mathrm{L}} \ll h_{\text {jump }}$ and $U_{\mathrm{S}}=U_{\mathrm{L}}$ if $h_{\mathrm{L}} \approx h_{\text {jump }}$. The jump altitude is presented in Figure $3 \mathrm{~d}$, and it is easily obtained from equation (4) by recognizing that $L_{S} \rightarrow \infty$ when $U_{\mathrm{L}}=h_{N} E_{\mathrm{cr}, \mathrm{L}}$, consequently $h_{\text {jump }}=h_{N} \ln \left(h_{N} E_{\mathrm{cr}, 0} / U_{\mathrm{L}}\right)$.
When $U_{\mathrm{L}} \ll h_{N} E_{\text {cr, } \mathrm{L}}$, equation (4) reduces to $L_{\mathrm{S}}=U_{\mathrm{S}} / E_{\text {cr, } \mathrm{L}}$. The condition $U_{\mathrm{L}} / E_{\mathrm{cr}, \mathrm{L}}=h_{N}$, approximating the jump altitude, points out that the streamer zone jumps to the ionosphere when its size becomes longer than the atmospheric scale height $h_{N}$.

[10] Note that for the same values of $h_{\mathrm{L}}$ and $U_{\mathrm{L}}$, the streamer zone of an upward negative leader is shorter than that of the positive one, as shown in Figure $3 \mathrm{a}$ for $U_{\mathrm{L}}=10$ MV. Consequently, the jump altitude is higher for negative leaders, as shown in Figure 3d.

\section{Vertical Structuring of GJs}

[11] Figures $2 \mathrm{c}$ and $2 \mathrm{~d}$ display the upward propagation as a function of time of two GJs observed by Pasko et al. [2002] and Soula et al. [2011] [see also da Silva and Pasko, 2012, Figure 1]. To model the GJ propagation, we assume (for simplicity) that a constant current $I=2.7$ A (Figure 2c) and $270 \mathrm{~A}$ (Figure $2 \mathrm{~d}$ ) flows through the leader stem. Initial current density $J=9.6 \times 10^{6} N^{2} / N_{0}^{2} \mathrm{~A} / \mathrm{m}^{2}$ is the same in both cases. In view of the discussion in section 2 , the difference in current is due to different initial stem radius $r_{c}=0.3 N_{0} / N$ $\mathrm{mm}$ and $3 N_{0} / N \mathrm{~mm}$, respectively. For these two values of current, the dependence $v_{\mathrm{L}}\left(I, h_{\mathrm{L}}\right)$ is obtained and a leader upward propagation is simulated by solving the equation $\mathrm{d} h_{\mathrm{L}} / \mathrm{d} t=v_{\mathrm{L}}\left(I, h_{\mathrm{L}}\right)[$ da Silva and Pasko, 2012]. For every position of the leader head $h_{\mathrm{L}}$, the size of the streamer zone is calculated from formula (4) assuming that $U_{\mathrm{S}}=U_{\mathrm{L}} / 2$, and that the leader potential is defined for a cylindric conductor elongating in an external uniform electric field, $U_{\mathrm{L}}=I / v_{\mathrm{L}} \mathcal{C}$, where the capacitance per unit length is $\mathcal{C} \approx 2 \pi \varepsilon_{0}=5.56 \times$ $10^{-11} \mathrm{~F} / \mathrm{m}$ [Bazelyan and Raizer, 2000, p. 62]. The shaded areas in Figures $2 \mathrm{c}$ and $2 \mathrm{~d}$ show the length of the streamer 
zone $L_{S}$ above the leader head, the lower boundaries of the shaded regions represent $h_{\mathrm{L}}$, while the upper boundaries $h_{\mathrm{S}}$. Leader potential varies within $0.8-30 \mathrm{MV}$ and 84-121 MV in Figures $2 \mathrm{c}$ and $2 \mathrm{~d}$, respectively. Consequently the streamer zone is shorter in Figure 2c.

[12] The conclusion to be drawn from Figures $2 \mathrm{c}$ and $2 \mathrm{~d}$ is that the strong acceleration in GJs is a consequence of their vertical structure. GJs initiate inside the thundercloud as a conventional intracloud lightning discharge. As demonstrated by Krehbiel et al. [2008], owing to the charge imbalance in thunderclouds (see also section 1), one or more lightning leaders can escape upward. The leader propagates upwards with a stable speed $\lesssim 10^{5} \mathrm{~m} / \mathrm{s}$, consistent to a current density of $\lesssim 10^{7} N^{2} / N_{0}^{2} \mathrm{~A} / \mathrm{m}^{2}$ in the leader stem (see Figures $2 \mathrm{a}$ and $2 \mathrm{~b}$ ). The leader is capable of bringing the high thundercloud potential $U_{\mathrm{L}}$ to upper altitudes [Raizer et al., 2006]. When the leader approaches the jump altitude (Figure 3d), the streamer zone expands causing the observed acceleration. During this stage the GJ speed is closer to that of fast streamers $\sim 10^{6}-10^{7} \mathrm{~m} / \mathrm{s}$ [e.g., Pasko, 2006, p. 259]. Results presented in Figures 2c and $2 \mathrm{~d}$ indicate that the initial leader stem radius (prior to channel contraction) should be larger than that of a single streamer, and more likely to be a few millimeters (scaled to ground pressure). Thus, the upward propagating GJ would carry a current of tens to hundreds of amperes, on the same order of magnitude as is reported in measurements [e.g., Cummer et al., 2009].

[13] The jump altitude is a useful parameter to trace the transition between leader and streamer portions of GJS during the leading jet phase. After the connection to the ionosphere, GJs exhibit a return stroke-like process [Kuo et al., 2009, Figure 5]. Owing to the high electrical current, $\sim 1 \mathrm{kA}$, flowing through the channel [Cummer et al., 2009, Figure 3] the leader portion may reach higher altitudes. However, a leader cannot bridge the gap between cloud and ionosphere, because streamer-to-leader transition is hindered at very low air densities. The air heating time exhibits a strong quadratic dependence on air density, $\tau_{\mathrm{h}}=$ $\tau_{\mathrm{h}, 0} N_{0}^{2} / N^{2}$ at near ground pressures. At very low air densities, where $\tau_{\mathrm{h}}$ is longer than the time scale for pressure equalization in the leader stem $\tau_{c}=r_{c} / c_{s}$ (where $c_{s}$ is the speed of sound), air heating is isobaric and occurs at slower rate, i.e., $\tau_{\mathrm{h}}>\tau_{\mathrm{h}, 0} N_{0}^{2} / N^{2}$ [da Silva and Pasko, 2012]. Therefore, for each set of initial conditions ( $I$ and $r_{c}$ ), there is an altitude above which streamer-to-leader transition can not occur. For example, modeling results indicate that, for $I=0.5 \mathrm{kA}$ and $r_{c}=0.3 N_{0} / N \mathrm{~mm}$, streamer-to-leader transition can not occur for above $\sim 70 \mathrm{~km}$ altitude. It takes a time $\tau_{\mathrm{h}} \simeq 1 \mathrm{~s}$ to significantly heat the air at $68 \mathrm{~km}$ altitude, longer than the duration of the whole GJ event, which is hundreds of milliseconds.

\section{Summary}

[14] In this letter we have reported simulation results on leader speeds, pointing out their dependence on current density in the leader stem, instead of total current as typically assumed in existing literature. We have discussed the dynamics of an upward propagating leader in a nonuniform atmosphere. We have demonstrated that the GJ acceleration can be understood as a consequence of the expansion of the leader streamer zone, as previously hypothesized. Therefore, the jump altitude may serve as a first-order estimate for the transition region between leader and streamer mechanisms in GJs.

[15] Acknowledgments. This research was supported by NSF AGS0652148 and AGS-0836391 grants to Pennsylvania State University.

[16] The Editor thanks two anonymous reviewers for their assistance in evaluating this paper.

\section{References}

Andreev, A. G., E. M. Bazelyan, M. U. Bulatov, I. P. Kuzhekin, L. M. Makalsky, D. I. Sukharevskij, and V. S. Syssoev (2008), Experimental study of the positive leader velocity as a function of the current in the initial and final-jump phases of a spark discharge, Plasma Phys. Rep., 34(7), 609-615, doi:10.1134/S1063780X0807009X.

Bazelyan, E. M., and Y. P. Raizer (2000), Lightning Physics and Lightning Protection, Inst. of Phys. Publ., Bristol, U. K.

Bazelyan, E. M., Y. P. Raizer, and N. L. Aleksandrov (2007), The effect of reduced air density on streamer-to-leader transition and on properties of long positive leader, J. Phys. D Appl. Phys., 40(14), 4133-4144, doi:10.1088/0022-3727/40/14/007.

Cummer, S. A., J. Li, F. Han, G. Lu, N. Jaugey, W. A. Lyons, and T. E. Nelson (2009), Quantification of the troposphere-to-ionosphere charge transfer in a gigantic jet, Nat. Geosci., 2(9), 617-620, doi:10.1038/ NGEO607.

da Silva, C. L., and V. P. Pasko (2012), Simulation of leader speeds at gigantic jet altitudes, Geophys. Res. Lett., 39, L13805, doi:10.1029/ 2012 GL052251.

Krehbiel, P. R., J. A. Riousset, V. P. Pasko, R. J. Thomas, W. Rison, M. A. Stanley, and H. E. Edens (2008), Upward electrical discharges from thunderstorms, Nat. Geosci., 1, 233-237, doi:10.1038/ngeo162.

Kuo, C.-L., et al. (2009), Discharge processes, electric field, and electron energy in ISUAL-recorded gigantic jets, J. Geophys. Res., 114, A04314, doi:10.1029/2008JA013791.

Lu, G., et al. (2011), Lightning development associated with two negative gigantic jets, Geophys. Res. Lett., 38, L12801, doi:10.1029/ 2011GL047662.

Pasko, V. P. (2006), Theoretical modeling of sprites and jets, in Sprites, Elves and Intense Lightning Discharges, NATO Sci. Ser. II, vol. 225, edited by M. Fullekrug, E. A. Mareev, and M. J. Rycroft, pp. 253-311, Springer, Heidelberg.

Pasko, V. P., M. A. Stanley, J. D. Mathews, U. S. Inan, and T. G. Wood (2002), Electrical discharge from a thundercloud top to the lower ionosphere, Nature, 416(6877), 152-154, doi:10.1038/416152a.

Popov, N. A. (2009), Study of the formation and propagation of a leader channel in air, Plasma Phys. Rep., 35, 785-793, doi:10.1134/ S1063780X09090074.

Raizer, Y. P., G. M. Milikh, and M. N. Shneider (2006), On the mechanism of blue jet formation and propagation, Geophys. Res. Lett., 33, L23801, doi:10.1029/2006GL027697.

Riousset, J. A., V. P. Pasko, P. R. Krehbiel, R. J. Thomas, and W. Rison (2007), Three-dimensional fractal modeling of intracloud lightning discharge in a New Mexico thunderstorm and comparison with lightning mapping observations, J. Geophys. Res., 112, D15203, doi:10.1029/2006JD007621.

Riousset, J. A., V. P. Pasko, and A. Bourdon (2010), Air-density-dependent model for analysis of air heating associated with streamers, leaders, and transient luminous events, J. Geophys. Res., 115, A12321, doi:10.1029/2010JA015918.

Soula, S., O. A. van der Velde, J. Montanya, P. Huet, C. Barthe, and J. Bór (2011), Gigantic jets produced by an isolated tropical thunderstorm near Réunion Island, J. Geophys. Res., 116, D19103, doi:10.1029/2010JD015581.

Su, H. T., R. R. Hsu, A. B. Chen, Y. C. Wang, W. S. Hsiao, W. C. Lai, L. C. Lee, M. Sato, and H. Fukunishi (2003), Gigantic jets between a thundercloud and the ionosphere, Nature, 423, 974-976, doi: $10.1038 /$ nature 01759 .

van der Velde, O., et al. (2010), Multi-instrumental observations of a positive gigantic jet produced by a winter thunderstorm in Europe, $J$. Geophys. Res., 115, D24301, doi:10.1029/2010JD014442. 